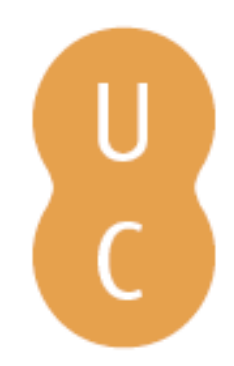

\title{
pompalina
}

\section{Émotion ressentie et simulée}

Autor(es): Hess, Ursula

Publicado por: Imprensa da Universidade de Coimbra; Les Éditions de L'IQRC (Les

Presses de l'Université Laval)

URL

persistente:

URI:http://hdl.handle.net/10316.2/32646

DOI:

DOI:http://dx.doi.org/10.14195/978-989-26-0805-1_6

Accessed : $\quad$ 26-Apr-2023 14:43:33

A navegação consulta e descarregamento dos títulos inseridos nas Bibliotecas Digitais UC Digitalis, UC Pombalina e UC Impactum, pressupõem a aceitação plena e sem reservas dos Termos e Condições de Uso destas Bibliotecas Digitais, disponíveis em https://digitalis.uc.pt/pt-pt/termos.

Conforme exposto nos referidos Termos e Condições de Uso, o descarregamento de títulos de acesso restrito requer uma licença válida de autorização devendo o utilizador aceder ao(s) documento(s) a partir de um endereço de IP da instituição detentora da supramencionada licença.

Ao utilizador é apenas permitido o descarregamento para uso pessoal, pelo que o emprego do(s) título(s) descarregado(s) para outro fim, designadamente comercial, carece de autorização do respetivo autor ou editor da obra.

Na medida em que todas as obras da UC Digitalis se encontram protegidas pelo Código do Direito de Autor e Direitos Conexos e demais legislação aplicável, toda a cópia, parcial ou total, deste documento, nos casos em que é legalmente admitida, deverá conter ou fazer-se acompanhar por este aviso.

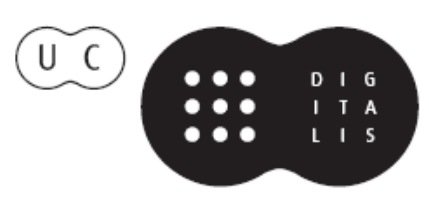


GILLES KIROUAC

Coordonnateur Scientifique

Cognition
et Émotions 
(Página deixada propositadamente em branco) 


\title{
GILLES KIROUAC
}

Coordonnateur Scientifique

\section{Cognition et Émotions}

\author{
AUTEURS \\ Klaus R. Scherer \& Janique Sangsue \\ Pierre Philippot \\ Dario Galati \\ Bernard Rimé \\ Pierre Gosselin \\ Ursula Hess \\ Arvid Kappas \& Jean Descôteaux \\ Pio E. Ricci Bitti \\ Pedro Luzes \\ Lise Fillion \\ Ramon Bayés \\ Coimbra - Imprensa da Universidade


COORDENAÇÃO EDITORIAL

Imprensa da Universidade de Coimbra

CONCEPÇÃO GRÁFICA

António Barros

PAGINAÇÃo

António Resende

[Universidade de Coimbra]

EXECUÇÃO GRÁFICA

G.C. - Gráfica de Coimbra, Lda.

Palheira - Assafarge - Apart. 3068

300I-453 Coimbra Codex

ISBN 972-8704-19-4

ISBN 2-7637-81।8-7

DEPÓSITO LEGAL 210430/04

(C) MAIO 2004, Imprensa DA UnIVERSIDAdE DE COIMBRA

\author{
OBRA PUBLICADA COM O PATROCINIO DO \\ INSTITUTO DE PSICOLOGIA COGNITIVA \\ FCT: FUNDAÇÃO PARA A CIÊNCIA E A TECNOLOGIA \\ MINISTÉRIO DA CIÊNCIA E DO ENSINO SUPERIOR \\ apolo do Programa Operacional CiênCIa, TeCnologia, Inovação \\ DO QUADRO COMUNITÁRIO DE APOIO
}




\section{Ursula Hess}

Département de Psychologie

Université du Québec à Montréal

\section{ÉMOTION RESSENTIE ET SIMULÉE}

\section{Introduction}

Cet article traitera des différences entre les émotions spontanées et les émotions simulées. Une telle distinction suscite la question de savoir si elle est utile sur le plan épistémologique et si oui, comment peut-on définir et distinguer les deux concepts ainsi proposés? Dans un premier temps, ces questions seront examinées et quelques réponses seront suggérées. Dans un deuxième temps, je présenterai quelques résultats empiriques qui portent sur cette différenciation et je tenterai d'intégrer ces informations dans un cadre qui considère les émotions simulées selon une perspective interactionnelle.

\section{Qu'est-ce qu'une émotion simulée?}

Afin de déterminer les caractéristiques des émotions simulées, il faudrait d'abord s'entendre sur la définition du terme émotion. Un grand nombre de définitions ont été proposées, dont plusieurs partagent des points communs. Récemment, les théories cognitives qui considèrent les émotions comme des produits d'un processus d'évaluation cognitive ont eu préséance dans la littérature (e.g. Scherer, 1986; Frijda, 1986; Lazarus, 1991). Toutefois, des positions fonctionnalistes ont aussi été ardemment défendues (e.g. Tomkins, 1962, 1963, 1991, 1993; Plutchik, 1962, 1980; Ekman, 1984; Izard, 1971, 1991). Ces deux approches s'entendent pour dire que les émotions sont caractérisées par des sensations subjectives, des réactions physiologiques, ainsi que des comportements verbaux et nonverbaux qui peuvent être ou non synchronisés. La majorité de ces définitions s'entendent sur le fait que les sensations subjectives sont une composante essentielle de l'émotion 
(Izard, 1990). De plus, il a été noté que les émotions se manifestent en réponse à certains types de stimuli (e.g. la tristesse comme réponse à une perte irrévocable, Lazarus, 1991). On pourrait donc dire que les émotions simulées sont des émotions qui apparaissent en l'absence de ces stimuli.

\section{Feeling rules}

Dans ce contexte, nous pouvons penser aux «feeling rules» proposées par Alice Hochschild (e.g., 1979; 1983). Cette dernière remarque que la société moderne exige de plus en plus que ses membres ressentent des émotions particulières dans certains contextes. Par exemple, elle note que plusieurs s'attendent à ce que dans le milieu de la santé les intervenants manifestent de la détresse et de la sympathie face au sort de leurs patients, tandis que d'autres exigent que les réceptionnistes et les agents de bord ressentent des émotions positives lors de leurs interactions avec leurs clients. Les «feeling rules» nécessitent que l'individu vive, et non seulement simule, l'émotion en question. Ainsi, la fatigue du passager ou la détresse du patient devraient être en soi des stimuli appropriés pour induire l'émotion culturellement appropriée et en conséquence leur affichage.

En effet, les demandes culturelles exigeant une certaine réaction émotionnelle face à une certaine classe des stimuli, cela devrait conduire automatiquement à l'expression d'une émotion accompagnée par l'état affectif correspondant. Étant donné que le sentiment est l'élément central pour la définition d'une émotion, l'émotion simulée ne pourrait donc être qu'une expression émotionnelle simulée.

\section{Règles d'affichage}

Les «display rules» ou «règles d'affichage des émotions», requièrent la simulation et la dissimulation des émotions. Ces règles d'affichage sont liées aux interactions sociales et guident l'affichage de certaines émotions dans des contextes précis. Ainsi, nous devrions ressentir de la joie lorsqu'on nous présente un cadeau, mais non à des funérailles. Cette idée que des normes sociales guident l'expression des émotions a été proposée parWundt (1903) et plus récemment, par Ekman et Friesen (1971). Ces auteurs avancent que 
ces règles sont spécifiques à chaque culture, mais que l'état affectif sous-jacent ne l'est pas. Ils présument alors, par example, que la majorité des individus ressentent de la colère face à un affront. Toutefois, dans certaines cultures ou sous-cultures, l'expression publique de cette colère pourrait être inhibée. D'autres chercheurs affirment plutôt que l'émotion comme telle est sociale et qu'elle est médiée par des connaissances culturelles. Chaque culture aurait ainsi ses propres déclencheurs d'émotions et pourrait définir les émotions de manière distincte (voir Markus \& Kitayama, 1994; Wierzbicka, 1994).

Des preuves existent pour valider ces deux perspectives. Des événements qui suscitent une émotion et les thèmes relationnels centraux proposés par Lazarus semblent être différents dans diverses cultures (e.g. Wierzbicka, 1995). De plus, les membres d'une même culture n'endossent pas tous l'expression de certaines émotions dans des contextes particuliers : par exemple, les femmes ont tendance à exprimer plus de peur que les hommes. En outre, les hommes et les femmes expriment une même émotion de manière différente. Par exemple, les femmes ont plus tendance à pleurer quand elles sont en colère que les hommes (Fischer, 1993). Ces derniers propos mettent en relief la dissociation possible entre l'état interne et l'affichage externe. Les contraintes culturelles qui demandent l'affichage d'une certaine émotion ou bien un certain type d'affichage d'émotions suscitent des expressions émotionnelles simulées. Dans une perspective interactionnelle, ces expressions simulées jouent le rôle d'émotions simulées.

L'expression des émotions simulées s'apprend tôt dans le processus de la socialisation de l'enfant. Les enfants peuvent utiliser les règles d'affichage dès leur jeune âge. Par exemple, Cole (1986) a démontré que des filles de 3 à 4 ans tentent de masquer leur déception lorsqu'elles reçoivent un cadeau indésirable. De même, Reissland et Harris (1991) ont trouvé que, même à 58 mois, des enfants essayent de cacher leur fierté dans certaines situations. Ces règles d'affichage peuvent être verbalisées par des enfants vers l'âge de six ans (e.g. Saarni, 1979).

\section{L'affichage d'émotions: symptômes ou signaux?}

La notion d'expressions émotionnelles simulées suppose qu'il existe une classe d'expressions qui représentent fiablement l'état émotif de l'émetteur. Ainsi, dans ce domaine de recherche, il y a une prémisse de base qui affirme qu'en 
temps normal, les expressions faciales émotionnelles agissent comme une représentation fidèle de l'état émotionnel de l'émetteur. Cette perspective date de l'Antiquité et Darwin (1872) déjà a décrit et souligné les similarités entre les diverses expressions émotionnelles chez l'humain et les animaux.

Récemment, toutefois, l'idée que les expressions émotionnelles faciales puissent être mieux comprises comme étant des communications d'intentions, modulées par la présence des autres et indépendantes de l'état émotionnel concomitant, a attiré l'attention de plusieurs chercheurs (Chovil, I99I; Fridlund, 1991; Fridlund, Kenworthy, \& Jaffey, 1992). Cette proposition est basée sur l'écologie comportementale qui affirme que - puisque les expressions faciales émotionnelles servent évidemment une fonction communicative et qu'on peut démontrer qu'elles peuvent être modifiées en présence d'un public l'explication voulant que les expressions faciales émotionnelles soient des symptômes d'un état affectif sous-jacent devient désuète.

Selon Fridlund et al. (1992), «l'effet d'auditoire» apporte un soutien empirique important à cette proposition, puisque ce phénomène souligne le caractère social de l'affichage émotionnel. Dans une série d'études, il a démontré, avec l'aide de ses collègues (Fridlund, 1991; Fridlund et al., 1992), que l'affichage d'expressions faciales négatives et positives subit une augmentation en présence d'un public réel ou imaginé. Fridlund, à partir de ses données, en vient à la conclusion que l'intensité des expressions faciales émotionnelles peut être prédite avec plus d'efficacité par le contexte social que par l'état affectif. II ajoute que ces résultats démontrent la nécessité de rejeter la notion «romantique» voulant que les expressions faciales soient une représentation fidèle et adéquate de l'état affectif sous-jacent.

La majorité des arguments principaux de Fridlund ne sont pas nouveaux. Le milieu de la recherche a reconnu, et ce depuis longtemps, que les expressions faciales peuvent être utilisées comme des symboles (Ekman, 118 1979), comme des interjections (Motley, 1993) et comme des signaux qui indiquent que l'individu comprend l'état émotionnel de son interlocuteur (Bavelas et al., 1986). En effet, il est évident que les expressions faciales, comme la plupart des messages, peuvent servir différentes fonctions en même temps. Cette proposition a généralement été véhiculée par ceux qui prétendent que les expressions faciales sont des symptômes d'un état affectif sous-jacent (e.g., Buck, 199I, Buck et al., 1992; Cacioppo, Bush, \& Tassinary, 1992; Hess, Kappas, \& Banse, 1995; Hess, Kappas, Kleck, McHugo, \& Lanzetta, 1989; Hess \& Kleck, 1990). 
Ainsi, le rôle des expressions faciales comme signaux d'interaction n'exclut pas leur rôle comme symptômes d'états émotionnels. De plus, il existe des données neurologiques qui montrent des différences de mécanismes entre les expressions faciales provoquées par un état émotionnel et celles qui sont produites volontairement. Plus particulièrement, les expressions faciales qui correspondent à un état affectif sous-ajacent semblent être innervées par des voies neuronales différentes que les expressions faciales simulées (voir Rinn, 1984, 1991). Par ailleurs, des résultats découlant d'études menées auprès des patients ayant une section du corps calleux démontrent que bien que les deux hémisphères puissent générer des expressions faciales spontanées, seule l'hémisphère gauche peut générer des expressions volontaires (Gazzaniga \& Smylie, 1990).

\section{L'effet d'auditoire réexaminé}

Nous avons illustré la fonction des expressions faciales émotionnelles comme représentations d'un état affectif et comme signal social (Hess, Banse, \& Kappas, 1995). Pour cela, nous avons reproduit l'expérience de Fridlund (|99|) en variant et l'aspect social du contexte et l'intensité du stimulus émotionnel, ainsi que la relation entre l'individu appelé à exprimer une émotion et son public. Les résultats indiquent que l'intensité de l'expression ne peut pas être prédite de manière satisfaisante par un seul de ces facteurs, mais plutôt par un mélange complexe des trois. Spécifiquement, nous avons trouvé que, lorsque les sujets regardaient des films qui variaient dans leur contenu humoristique soit en présence d'un copain, soit lorsque le copain fait la même tâche, mais dans une autre salle, ou lorsque le copain fait une autre tâche dans une salle différente, l'activité du Orbicularis Occuli (qui indique la présence d'un sourire) variait en fonction et du contenu humoristique et en fonction du degré de présence du «public». Ainsi, les sujets souriaient plus en regardant le film plus drôle que lorsqu'ils regardaient le film plus sérieux. De plus, les sujets souriaient plus souvent lorsqu'ils étaient en compagnie de leur ami que lorsque ce dernier travaillait à une tâche différente dans une autre salle. II n'y avait pas de différences notables lorsque le compagnon du sujet était un étranger. Alors, dans ce contexte, les expressions faciales ont servi de signaux pour communiquer le degré d'amusement de l'émetteur à son ami, en plus de représenter son état émotionnel. 


\section{Définition des expressions émotionnelles simulées}

Toutefois, les réserves émises par Fridlund (199|) concernant les expressions émotionnelles spontanées et simulées doivent être prises en considération. J'ai déjà proposé l'idée (Hess \& Kleck, 1990) qu'une simple séparation dichotomique des expressions en deux catégories, soit les expressions «senties» et les expressions «fausses», classification courante dans la littérature portant sur ce domaine (e.g., Ekman \& Friesen, 1982), ne rend pas justice à la complexité du phénomène. Au contraire, les expressions faciales émotionnelles spontanées et les expressions faciales émotionnelles voluntaires peuvent être considérées comme des extrêmes d'un même continuum.

Dans ce continuum, les expressions spontanées correspondent à l'état émotionnel de la personne et ne subissent pas ou peu de contrôle. Par contre, les expressions simulées sont des expressions qui ont une composante volontaire. Ces expressions peuvent être soit des expressions correspondant à l'état émotionnel de la personne, mais qui sont augmentées ou diminuées en fonction des contraintes de la situation, ou soit des expressions qui ne correspondent pas du tout à l'état émotionnel de l'individu.

Par conséquent, les expressions faciales émotionnelles peuvent être situées dans un espace bi-dimensionnel où une des dimensions représente l'état émotionnel de l'émetteur et l'autre dimension, l'émotion exprimée.

Les expressions émotionnelles spontanées reflètent la valence et l' intensité de l'état affectif de l'émetteur. Les expressions émotionnelles simulées soit peuvent varier en intensité, mais non en valence selon l'état affectif de l'émetteur (i.e. des expressions inhibées ou exagérées), soit, peuvent ne pas correspondre à l'état affectif de l'émetteur. Dans ce dernier cas, on parle de dissimulation.

L'affichage d'émotions simulées et la tromperie volontaire ne sont pas des termes équivalents. La tromperie volontaire, par définition, suppose que l'émetteur veuille tromper le récepteur, mais la simulation peut se produire dans d'autres circonstances. Principalement, en suivant les règlements d'affichage, les gens peuvent vouloir augmenter leur estime de soi. Ainsi, en masquant une émotion (e.g., la colère), un individu peut vouloir démontrer un comportement approprié, sans nécessairement vouloir consciemment tricher l'autre. Cette inhibition peut même se produire lorsque l'individu est seul et ne se fait pas observer. Dans cette circonstance, c'est cette conscience de vouloir bien agir qui motive le comportement. 
Par ailleurs, une école de pensée récente a ravivé la question entourant les émotions inconscientes, c'est-à-dire, les émotions qui pourraient être réprimées de la conscience rationnelle tout en laissant des traces dans le domaine nonverbal. Dans ces cas-là, la distinction entre la dissimulation volontaire - bien que non réussie - et la répression, est difficile à faire (Ekman, 1992; Lazarus, 1995). De plus, dans la mesure où l'état affectif subjectif est jugé comme étant la composante principale de l'émotion (voir Izard, 1990), le raisonnement devient même tautologique.

Aussi, certaines expressions volontaires peuvent se produire spontanément, comme c'est le cas des réactions surapprises qui s'apparentent à un réflexe. Un exemple de ce type de réaction serait le sourire émis pour saluer une connaissance. Ce sourire ne correspond pas nécessairement à un état réel de joie, mais fait plutôt partie d'un protocole social. Par conséquent, il est rarement interprété comme étant un signe indiquant l'état affectif de l'émetteur.

En résumé, pour classifier les expressions émotionnelles simulées comme étant une tromperie, il faut prendre en considération la motivation et l'état subjectif de l'émetteur et les attributions du receveur. C'est donc dire que, dans des situations où l'émetteur et le receveur partagent les mêmes règles culturelles s'entendent sur l'exactitude de l'expression compte tenu de la situation et reconnaissent l'expression comme un symbole plutôt qu'une représentation d'un état émotif (comme c'est le cas pour le sourire de salutation), alors il n'y a pas tromperie.

Il faut noter que le rôle actif de l'observateur dans ce processus a été largement ignoré par les chercheurs malgré le fait que la notion d'émotions simulées suppose implicitement la présence d'un observateur.

\section{L'encodage et le décodage d'expressions émotionnelles intentionnelles}

L'encodage d'expressions faciales émotionnelles subit l'influence d'une série de facteurs, incluant les contraintes sociales telles les règles d'affichage. Certains résultats émanant de notre laboratoire semblent indiquer que le décodage des expressions faciales émotionnelles peut subir des influences similaires.

La majorité des études qui traitent des expressions faciales intentionnelles se concentrent sur l'encodage et le décodage de messages trompeurs plutôt que sur les expressions émotionnelles volontaires comme telles. En ce qui a trait à l'encodage des expressions émotionnelles intentionnelles, la plupart 
des recherches ont porté sur les sourires. Duchenne avait déjà noté, en 1863, que les sourires spontanés sont caractérisés par une activité du muscle Orbicularis Oculi qui produit des rides autour des yeux. Ces résultats ont été confirmés par Ekman et ses collègues (Ekman \& Friesen, 1982; Ekman, Friesen, \& O'Sullivan, 1988; Ekman, Davidson, \& Friesen, 1990). Hess et al. (1989) ont aussi trouvé des différences entre les sourires spontanés et intentionnels en utilisant l'électromyographie faciale. De plus, il a été remarqué que les expressions volontaires sont plus asymétriques que les expressions spontanées (Hagar \& Ekman, 1985). Enfin, les sourires spontanés et volontaires ont des parcours temporels différents (Bugenthal, 1986; Ekman \& Friesen, 1982; Weiss, Blum, \& Gleberman, 1987; Hess \& Kleck, 1990). En somme, il existe de résultats démontrant que les sourires spontanés et délibérés diffèrent. Par conséquent, on pourrait s'attendre à ce que les décodeurs puissent distinguer les expressions de joie qui sont simulées de celles qui sont spontanées.

Toutefois, il n'en est pas ainsi. En général, les chercheurs ont remarqué que les gens ne sont pas très habiles lorsque vient le temps d'identifier les expressions simulées. Dans une importante étude, Ekman et O'Sullivan (1991) a tenté de vérifier cette capacité chez des gens de différentes professions pour qui de bonnes habiletés de décodage peuvent être d'une grande utilité (e.g., juges, policiers, douaniers, etc.). Ils ont trouvé une moyenne de décodage exact autour de 50\% - c'est-à-dire au niveau attendu pour des jugements effectués au hasard.

Sur la base de ces résultats, on pourrait soutenir que les différences objectives entre les expressions spontanées et simulées sont tout simplement trop faibles ou rapides pour être détectées. Toutefois, Ekman et O'Sullivan (1991) a trouvé des taux de réussite beaucoup plus élevés pour deux groupes d'individus, soit les agents de la CIA comptant entre 5 et I 5 années d'expérience et les enfants, surtout ceux provenant de familles abusives. Ces résultats suggèrent que (a) les expressions émotionnelles spontanées et intentionnelles diffèrent de manière objective, (b) ces différences sont observables en principe et (c) ces différences ne sont généralement pas observées ou interprétées.

Nous avons étudié davantage ce phénomène (Hess \& Kleck, 1990, 1994). Dans une première étude, nous avons demandé à des sujets de faire plusieurs tâches qui étaient construites de façon à provoquer des expressions faciales spontanées ou posées de joie ou de dégoût. En se basant sur le 
FACS (Ekman \& Friesen, 1978), le temps du début, le temps de la fin, et le temps à l'apex (sommet) furent observés pour chaque épisode. Puisqu'une seule expression peut avoir plusieurs débuts et fins, le nombre de phases fut aussi mesuré. Les résultats démontrent que la régularité des expressions volontaires et spontanées varie. Les expressions spontanées sont relativement coulantes et régulières, tandis que l'intensité des expressions volontaires démontre plus de pauses et de changements en escalier.

Dans une seconde étude, les sujets devaient regarder 60 expressions de joie et de dégoût et tenter de déterminer pour chaque vignette si l'expression était spontanée ou volontaire. À la suite de l'expérience, les sujets devaient rapporter les indices particuliers qu'ils avaient utilisés pour distinguer les deux types d'expressions. Ces indices, de même que les signaux qui avaient auparavant été identifiés comme étant des indicateurs valides, ont été mesurés objectivement.

Des modèles par équations structurales ont été utilisés afin de vérifier si (a) les scores de réussite des sujets étaient liés aux indicateurs qu'ils ont dit utiliser et (b) si ces indicateurs étaient valides. Les résultats ont démontré que (a) les sujets ont rapporté adéquatement les indices qu'ils ont employés pour prendre leur décision et (b) la majorité de ces indicateurs n'aident pas à discriminer de manière valide les expressions spontanées des expressions volontaires. Ainsi, les sujets n'ont pas pu bien faire la différence entre les expressions spontanées et volontaires et ce piètre niveau de succès vient de ce qu'ils ont constamment employé des indicateurs non valables.

\section{Le rôle du décodeur}

Les données mentionnées ci-haut suggèrent que la majorité des gens ont des croyances erronées concernant les signaux qui permettent de distinguer les deux types d'expressions. Ceci est assez étonnant si l'on constate que deux catégories de sujets étudiés par Ekman et O'Sullivan (|99|) ont été capables de faire cette distinction.

Par conséquent, comme l'a suggéré Kraut (1980), nous devons donc supposer que les adultes ne sont généralement pas motivés à détecter les expressions faciales fausses. Ce chercheur affirme que des interactions harmonieuses dépendent de la volonté des gens d'accepter les messages au pied de la lettre. Cette proposition est appuyée par un phénomène 
souvent observé: les individus ont tendance à considérer la majorité des messages comme étant véridiques (Zuckerman, DePaulo, \& Rosenthal, 1981). De plus, d'autres résultats indiquent que des sujets souffrant d'un niveau élevé d'anxiété sociale sont beaucoup moins habiles à détecter la tromperie que des sujets qui ont des faibles niveaux d'anxiété sociale (DePaulo \& Tang, 1994).

II se peut donc que les observateurs utilisent des «normes de décodage» pour guider leur interprétation des expressions faciales émotionnelles. Ces règles permettent à l'individu d'ignorer les signes qui rendent possibles l'identification des expressions volontaires. Ces normes de décodage incorporent les connaissances sociales sur l'exactitude de certaines expressions faciales, tout comme le font les lois d'affichage. En fait, les normes de décodage peuvent simplement être considérées comme l'envers des lois d'affichage. Ainsi, l'encodeur utilise les lois d'affichage pour modifier ses expressions. II se fait aider par l'observateur qui a tendance à décoder l'émotion appropriée plutôt qu'une autre.

Certains résultats trouvés dans notre laboratoire ont appuyé l'existence de ces normes d'affichage et de décodage par rapport aux expressions faciales d'émotions chez les hommes et les femmes. Pendant l'expérience, les sujets ont regardé 24 paires de dessins illustrant des expressions faciales émotionnelles. Chaque expression était associée à un profil facial féminin ou masculin. Les données indiquent que, pour la colère et la tristesse, la même expression émotionnelle associée à un profil féminin était jugée comme étant plus intense que pour les profils masculins. Pour la joie, le contraire s'est produit : les expressions de joie associées à un profil masculin étaient jugées comme étant plus intenses que pour les profils féminins. Ces résultats démontrent l'influence des connaissances des rôles sociaux sur le décodage. Vu qu'une femme est censée exprimer la colère soit moins fortement qu'un

124 homme, soit d'une manière différente (en pleurant), une même expression de colère est interprétée comme indiquant une colère moyenne chez un homme, mais une colère forte chez une femme.

\section{Conclusion}

En conclusion, la notion d'émotion simulée devrait être remplacée par la notion de l'affichage émotionnel simulé. La simulation des émotions peut se situer dans un contexte de tromperie. Par ailleurs, les règles sociales 
gouvernant les interactions de tous les jours demandent l'affichage de certaines émotions dans certaines situations. La prévalence de ces demandes ainsi que leur importance sont soulignées par le fait que même les enfants en bas âge savent s'y conformer. Dans une perspective qui accorde au décodeur un rôle actif dans les processus interactionnels, le faible taux de réussite dans le décodage des expressions simulées est interprété comme résultant d'un partage de ces règles sociales.

\section{RÉFÉRENCES}

Bavelas, J. B., Black, A., Lemery, C. R., \& Mullett, J. (1986). "I show how you feel": Motor mimicry as a communicative act. Journal of Personality and Social Psychology, 50, 322-329.

Buck, R. (1991). Social functions in facial display and communication: A reply to Chovil and others. Journal of Nonverbal Behavior, 15, I55-161.

Buck, R., Loslow, J. I., Murphy, M. M., \& Costanzo, P. (1992). Social facilitation and inhibition of emotional expression and communication. Journal of Personality and Social Psychology, 63, 962-968.

Bugenthal, D. B. (1986). Unmasking the "polite smile": Situational and personal determinants of managed affect in adult-child interaction. Personality and Social Psychology Bulletin, 12 , 7-16.

Cacioppo, J.T., Bush, L. K., \& Tassinary, L. G. (1992). Microexpressive facial actions as a function of affective stimuli: Replication and extension. Personality and Social Psychology Bulletin, $18,515-526$.

Chevalier-Skolnikoff, S. (1973). Facial expression of emotion in nonhuman primates. In P. Ekman (Ed.), Darwin and facial expression (pp. I I-83). New York, NY: Academic Press.

Chovil, N., \& Fridlund, A. J. (1991). Why emotionality cannot equal sociality: A reply to Buck. Journal of Nonverbal Behavior, 15, 163-167.

Cole, P. M. (1986). Children's spontanous control of facial expressions. Child Development, 57, 1309-1321.

Darwin, C. (1872/1965). The expression of the emotions in man and animals. Chicago: The University of Chicago Press. (Originally published, 1872).

DePaulo, B. M., \& Tang, J. (1994). Social anxiety and social judgement: The example of detecting deception. Journal of Research in Personality, 28, 142-153.

Ekman, P. (1979). About brows: Emotional and conversational signs. In K. F. M. von Cranach W. Lepenies, \& D. Ploog (Ed.), Human ethology (pp. 169-249). Cambridge: Cambridge University Press.

Ekman, P. (1984). Expression and the nature of emotion. In K. R. Scherer \& P. Ekman (Eds.), Approaches to emotion (pp. 319-344). Hillsdale, NJ: Erlbaum.

Ekman, P. (1992). An argument for basic emotions. Cognition and Emotion, 6, 169-200.

Ekman, P., Davidson, R. J., \& Friesen, W.V. (1990). The duchenne smile: Emotional expression and brain physiology II. Journal of Personality and Social Psychology, 58, 342-353.

Ekman, P.. \& Friesen, W.V. (197I). Constants across cultures in the face and emotion. Journal of Personality and Social Psychology, 17, 124-129. 
Ekman, P. \&. F., W.V. (1978). The Facial Action Coding System: A technique for the measurement of facial movement. Palo Alto, CA: Consulting Psychologists Press.

Ekman, P., \& Friesen, W.V. (1982). Felt, false, and miserable smiles. Journal of Nonverbal Behavior, 6. 238-252.

Ekman, P., Friesen, W. V., \& O'Sullivan, M. (1988). Smiles when lying. Journal of Personality and Social Psychology, 54, 4I4-420.

Ekman, P., \& O'Sullivan, M. (1991). Who can catch a liar? American Psychologist, 46, 913-920.

Fischer, A. (1993). Sex differences in emotionality: Fact or Stereotype? Feminism \& Psychology, 3, 303-318.

Fridlund, A. J. (|99|). The sociality of solitary smiling: Potentiation by an implicit audience. Journal of Personality and Social Psychology, 60, 229-240.

Fridlund, A. J., Kenworthy, K. G., \& Jaffey, A. K. (1992). Audience effects in affective imagery: Replication and extension to dysphoric imagery. Journal of Nonverbal Behavior, 16 (3), 191 -211 .

Frijda, N. (1986). The emotions. Cambridge: Cambridge University Press.

Gazzaniga, M. S., \& Smylie, C. S. (1990). Hemispheric mechanisms controlling voluntary and spontaneous facial expressions. Journal of Cognitive Neuroscience, 2, 239-245.

Hager, J. C., \& Ekman, P. (1985). The asymmetry of facial actions is inconsistent with models of hemispheric specialization. Psychophysiology, 22, 307-317.

Hess, U., Kappas, A., \& Banse, R. (1995). The intensity of facial expressions is determined by underlying affective state and social situation. Journal of Personality and Social Psychology, 69, 280-288.

Hess, U., Kappas, A., McHugo, G. J., Kleck, R. E., \& Lanzetta, J. T. (1989). An analysis of the encoding and decoding of spontaneous and posed smiles: The use of facial electromyography. Journal of Nonverbal Behavior, 13(2), 121-137.

Hess, U., \& Kleck, R. E. (1990). Differentiating emotion elicited and deliberate emotional facial expressions. European Journal of Social Psychology, 20, 369-385.

Hess, U., \& Kleck, R. E. (1994). The cues decoders use in attempting to differentiate emotion elicited and posed facial expressions. European Journal of Social Psychology, 24, 367-38I.

Hochschild, A. (1983). The managed heart. Berkely: University of California Press.

Hochschild, A. R. (1979). Emotion work, feeling rules, and social structure. American journal of Sociology, 85(3), 55I-573.

Izard, C. E. (197|). Human emotions. New York: Plenum.

Izard, C. E. (1990). Facial expressions and the regulation of emotions. Journal of Personality and Social Psychology, 58, 487-498.

126 Izard, C. E. (1991). The psychology of emotions. New York: Plenum,

Kraut, R. E. (1980). Humans as lie detectors: some second thoughts. Journal of Communication, 30, 209-216.

Lazarus, R. R. (199|). Emotion and adaptation. New York: Oxford University Press.

Lazarus, R. S. (1995). Vexing research problems inherent in cognitive mediational theories of emotion - and some solutions. Psychological Inquiry, 6, 183-196.

Markus, H. R., \& Kitayama, S. (1994). The cultural construction of self and emotion: Implications for social behavior. In S. Kitayama \& H. R. Markus (Eds.), Emotion and culture: Empirical studies of mutual influence Washington, DC: American Psychological Association.

Motley, M. T. (1993). Facial affect and verbal context in conversation: Facial expression as interjection. Human Communication Research, 20, 3-40. 
Plutchik, R. (1962). The emotions: Facts, theories and a new model. New York, NY: Random House.

Plutchik, R. (1980). Emotion: A psychoevolutionary synthesis. New York, NY: Harper \& Row.

Reissland, N., \& Harris, P. (199I). Children's use of display rules in pride-eliciting situations. British Journal of Developmental Psychology, 9, 431-435.

Rinn, W. E. (1984). The neuropsychology of facial expression: A review of the neurological and psychological mechanisms for producing facial expressions. Psychological Bulletin, 95, 52-77.

Rinn, W. (1991). Neuropsychology of facial expression. In R. S. Feldman \& B. Rimé (Eds.), Fundamentals of nonverbal behavior (pp. 3-30). Cambridge: Cambridge University Press.

Saarni, C. (1979). Children's Understanding of display rules for expressive behavior. Developmental Psychology, 15, 424-429.

Scherer, K. R. (1986). Vocal affect expression: A review and a model for future research. Psych. Bull., 99(2), 143-165.

Tomkins, S. S. (1962). Affect, imagery, conciousness: The positive affects. New York: Springer.

Tomkins, S. S. (1963). Affect, imagery, conciousness: The negative affects. New York: Springer.

Tomkins, S. S. (199|). Affect, imagery, conciousness: Anger and fear. New York: Springer.

Tomkins, S. S. (1993). Affect, imagery, conciousness: Cognition - Duplication and transformation of information. New York: Springer.

Weiss, F., Blum, G.S., \& Gleberman, L. (1987). Anatomically based measurement of facial expressions in simulated versus hypnotically induced affect. Motivation and Emotion, II, 67-81.

Wierzbicka, A. (1994). Emotion, language, and cultural scripts. In S. Kitayama \& H. R. Markus (Eds.), Emotion and culture: Empirical studies of mutual influence Washington, DC: American Psychological Association.

Wierzbicka, A. (1995). The relevance of language to the study of emotions. Psychological Inquiry, 6, 248-252.

Wundt, W. (1903). Grundzüge der physiologischen Psychologie. Leipzig: Wilhelm Engelmann.

Zuckerman, M., DePaulo, B. M., \& \& Rosenthal, R. (198I). Verbal and nonverbal communication of deception. In L. Berkowitz (Ed.), Advances in experimental social psychology (pp. 1-59). New York: Academic Press. 
Série

Investigaçāo

Coimbra

Imprensa da Universidade

Les Presses de L'Université Laval

Les Editions de L'IORC

2004 